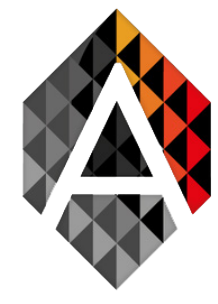

\title{
The Impact of IEEE 802.11 Contention Window on The Performance of Transmission Control Protocol in Mobile Ad-Hoc Network
}

\author{
Iqtidar Ali ${ }^{1}$, Tariq Hussain ${ }^{2}$, Kamran Khan ${ }^{1}$, Arshad Iqbal ${ }^{1}$ \\ and Fatima Perviz ${ }^{1}$ \\ 1 The University of Agricultural Peshawar, Pakistan \\ 2 School of Computer Science and Information Engineering, Zhejiang Gongshang \\ University China \\ iqtidar@aup.edu.pk, uom.tariq@gmail.com, aiqbal@aup.edu.pk, kamrankhan_47@yahoo. \\ com, faaatimakhaaan31@gmail.com
}

KEYWORD

MANET, $C W$, TCP, MAC. IEEE 802.11

\section{ABSTRACT}

A Mobile Ad-hoc Network (MANET) is a group of nodes connected via adhoc fashion for communicating with each other through a wireless interface. The communication among the nodes in such a network takes place by using multi-hop in the absence of fixed infrastructure. TCP faces some hurdles and complexities in multi-hop ad-hoc networks particularly congestion and route failures. The incompatibility between the MAC and TCP are previously noticed by the research community. This research focuses on the impact of the MAC layer contention window on TCP in MANET by using variation in network density and velocity of nodes respectively. Simulation has been carried out to quantify and analyze the impact of Contention Window $(C W)$ sizes that affect the performance of TCP by using the NS-2 simulator. The impact of $C W$ is investigated on TCP in multi-hop networks utilizing performance evaluation parameters i.e. average delay, average packet drops, and average throughput, the CW Min performance is better than as compared to other contention windows.
Iqtidar Ali, Tariq Hussain, Kamran Khan, Arshad lqbal and Fatima Perviz

The Impact of IEEE 802.11 Contention Window on The Performance of Transmission Control Protocol...
ADCAIJ: Advances in Distributed Computing and Artificial Intelligence Journal Regular Issue, Vol. 9 N. 3 (2020), 29-48 eISSN: 2255-2863 - https://adcaij.usal.es Ediciones Universidad de Salamanca - CC BY-NC-ND 


\section{Introduction}

Wireless networks can be categorized broadly into two types and the difference between them is not much as it seems. The first and the major type which is used the most in today's world is the infrastructure network having a backbone of a wired network (Assasa et al., 2018). In a wired network, the wireless nodes act like bridges. The wireless nodes in this type of network are known as base stations. Cellular phones network where the connectivity is based on the best signal quality is the best example of the wireless networks. As the strength of the signal goes weak a hand-off is performed by the phone and switch to a new base station within its range with better quality signals. The hand-off process is very fast and seamless for the network user. The second major type of wireless networks is known as the Ad-hoc approach which is independent and does not rely on some type of fixed and immobile infrastructure (Assasa et al., 2018; Bheemalingaiah et al., 2017). The nodes in this type of network are not stationary, they are mobile and the connectivity is also dynamically and arbitrarily. The wireless nodes in this type of network can act as a router and host at the same time and can also take an active part in the creation and maintenance of routes in the network (Khawas \& Gautam, 2017). The nodes in an ad-hoc network that are far away from each other and or not within the transmission range can communicate with each other through intermediate nodes (node acts a router along the path). The network topology may change dynamically and arbitrarily because of the mobility of nodes, the freedom of mobility allowing the nodes to leaving and joining the network at any instance (Kanellopoulos, 2019). The positive aspects of these networks are that they can be placed anywhere with ease without any infrastructure present in advance. The cost of deployment is low and does not require any administration except for the initial configuration. These networks are becoming popular increasingly in many areas like military battlefields, outdoor business meetings, disaster recovery and rescue operations, environmental protection agencies, and entertainment. Besides the advantages, there are some limitations of ad-hoc networks like limited bandwidth, dynamism in topologies, quality of the links, energy-constrained, and variations in capabilities are some constraints that harm its performance (Pokhrel et al., 2017). The Architecture of MANET is shown in figure 1.

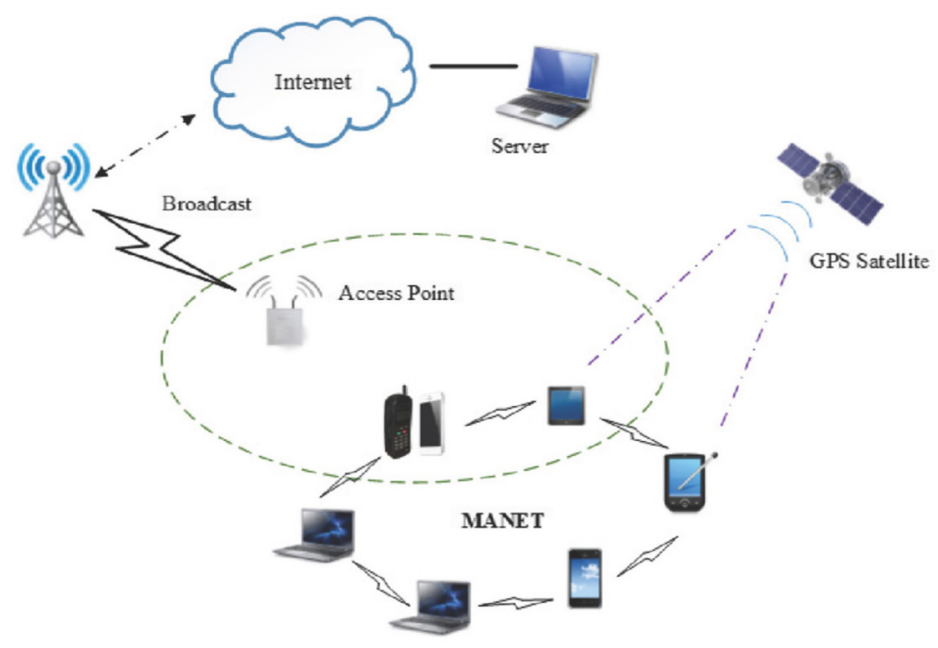

Fig 1. Architecture of MANET.

Iqtidar Ali, Tariq Hussain, Kamran Khan, Arshad lqbal and Fatima Perviz

The Impact of IEEE 802.11 Contention Window on The Performance of Transmission Control Protocol...
ADCAIJ: Advances in Distributed Computing and Artificial Intelligence Journal Regular Issue, Vol. 9 N. 3 (2020), 29-48 eISSN: 2255-2863 - https://adcaij.usal.es Ediciones Universidad de Salamanca - CC BY-NC-ND 
The dominant technology which provides high-speed access to the network in today's world is IEEE 802.11 [11]. In 802.11 CSMA/CA is a mechanism used for accessing the underlying channel. The two dominant and most popular medium access protocols used in 802.11 is DCF and PCF. IEEE 802.11 further consists of two phases for accessing the channel and transmission of data i.e. the basic access mode which is known as a two-way handshake while the other one is a four-way handshake normally known as Request to Send/Clear to Send (RTS/CTS) mechanism (Pokhrel et al., 2017). The basic model operates by using two-way handshake mechanism data is sent and wait for the acknowledgment to guarantee the successful transmission of data. This method is adopted when the data packet is small. The four-way handshake or RTS/CTS mechanism is adopted for exchanging data in an adhoc fashion where the size of data packets is large. The RTS/CTS packets are exchanged before data packets to analyze the medium whether it is free, or another transmission is in progress. There are two purposes behind the use of RTS/CTS exchange to direct and organize the transmission of data between transmitter and receiver and to declare the interval of time for which the ongoing communication between two entities will be held (Chu, 2005). A node that wants communicates using 802.11 CSMA/ CA in ad-hoc networks listen to the channel whether it is free or not. The packet is transmitted when the channel is in an idle state or wait for some time (contention period) if found busy. The time of contention is the time interval when two nodes are transferring data in a shared medium, the amount of time is distributed equally among nodes in a shared medium during transmission. The waiting amount of time is defined by DIFS in IEEE 802.11 standards (Babu \& Geethanjali). The incompatibility between TCP and MAC layer is another major issue that can impact the performance of MANET up-to some extent (Banerji \& Chowdhury, 2013). Another reason for performance degradation is the shared medium among multiple nodes results in contention at the MAC layer, mostly caused by the hidden nodes phenomenon. Packets lost due to buffer overflow can also affect TCP performance in MANET (Bhople \& Tijare, 2011).

\subsection{Transmission Control Protocol (TCP)}

TCP is the most widely used protocol nowadays on the Internet is a reliable connection-oriented byte stream protocol originally designed for traditional wired networks with stationary hosts. It is considered as the most trustworthy and the most widely deployed protocol that is well-suited with nearly all the protocols and applications on the Internet (Ali et al., 2020). However, the existing TCP which was initially proposed for infrastructure wired and laterally for fixed wireless networks may not fit in multi-hop ad-hoc because in a wired environment every type of packet loss is treated as congestion (Nithya et al., 2014). While in wireless and specifically in multi-hop ad-hoc networks wireless links and channels are affected by several other reasons like different types of channel errors, mobility which results in loss of data. The packets may be dropped to some other reason in such a network while TCP will invoke its congestion control algorithm which will dramatically degrade the performance of the network (Purniya \& Rai). Packet losses in multi-hop ad-hoc networks may be dropped due to route failures, medium contention, and channel errors so the assumption of these as congestion is not valid issues furthermore. A separate mechanism should be required for ad-hoc networks which could differentiate and treat contention, congestion, and channel errors separately to enhance and increase the efficiency of ad-hoc networks specifically in terms of throughput and packet delivery ratio. The coupling of the problems and the grasping properties of TCP could lead to instability which results in a decrease of throughput and excessive long delays in the network (Zhai et al., 2006).

Iqtidar Ali, Tariq Hussain, Kamran Khan, Arshad lqbal and Fatima Perviz

The Impact of IEEE 802.11 Contention Window on

The Performance of Transmission Control Protocol...
ADCAIJ: Advances in Distributed Computing and Artificial Intelligence Journal Regular Issue, Vol. 9 N. 3 (2020), 29-48 eISSN: 2255-2863 - https://adcaij.usal.es Ediciones Universidad de Salamanca - CC BY-NC-ND 


\subsection{Our Contribution}

In wireless local area networks,s the medium of communication is shared among mobile nodes with having limited range using Distributed Coordination Function (DCF). The performance of DCF relies on the Contention Window $(\mathrm{CW})$ adaption and the backoff strategy. $\mathrm{CW}$ is the major parameter of DCF and backs off the algorithm. Several features are introduced by the contention window mechanism in IEEE 802.11 Data Coordination function (DCF) which is the underlying protocol for accessing the shared wireless media multi-hop networks. The main problem is associated with the Contention Window $(\mathrm{CW})$. The TCP segments coming at various rates are split into small Medium Access Control (MAC) layer packets with a differed interval. To restrict the range of contention window size, a minimum contention window ( $\mathrm{CW}$ Min) and maximum contention window ( $\mathrm{CW}$ Max) is defined. The contention window is reset to the minimum contention window with success and doubling with collision. Resetting the contention window to its initial minimum contention window can cause severe degradation in performance when the minimum window is too small. The focus of this research study will be to find out the impact of the IEEE 802.11 DCF (Contention Window) on the performance of TCP in MANET. The contention window may be reset to some new window size according to the offered network load.

- To study and investigate the impact of variable contention window size on the performance of TCP.

- To evaluate the performance of TCP with varying incremental contention windows based on metrics, i.e. Average Throughput, Average Delay, and Average Packet Drop.

\subsection{Motivation}

The fixed contention resolution technique deployed in the primary BEB algorithm is not suitable for highly dynamic ad-hoc networks. It is essential to emphasize an effective algorithm based on dynamic contention window adjustment based on load. Every node in the network needs to be forced for keeping track of the network status at any time. The extra overhead results in the degradation of network performances. This motivates to development of an effective contention calculation mechanism suitable for such networks and reduces the control overhead and complex computation.

\subsection{Scope and Significance}

Applications of MANET is a diverse domain, ranging from small static networks operated by low power sources to large scale highly dynamic mobile networks. Significant instances comprised of forming survivable, effective, dynamic communication for military battlefields disaster relief operations, rescue/emergency operations, conferences, fault-tolerant sensor grids, intelligent transportation systems, environmental control, patient monitoring, smart homes, and numerous security-sensitive applications. Most of these domains efficient and reliable communication. The quick and probably short-term establishment of military communications in unknown and hostile environments, similarly for treating the patient at mobile locations also needs a quick response. The nodes in the discussed environment are autonomous and have the equipment for radio interface having distinct transmission/ reception capabilities which result in asymmetric links. The communication takes place among nodes by using a layered architecture. In the layered protocol stack, every layer is independent in its functionality, each layer deals only with its own ignoring the impacts on other layers present at the stack. In multi-hop networks keeping the above characteristics in mind, the functionalities of the Medium

Iqtidar Ali, Tariq Hussain, Kamran Khan, Arshad lqbal and Fatima Perviz

The Impact of IEEE 802.11 Contention Window on

The Performance of Transmission Control Protocol...
ADCAIJ: Advances in Distributed Computing and Artificial Intelligence Journal Regular Issue, Vol. 9 N. 3 (2020), 29-48 eISSN: 2255-2863 - https://adcaij.usal.es Ediciones Universidad de Salamanca - CC BY-NC-ND 
Access Control (MAC) layer and transport layer TCP is of much significance. As noticed by this research study that the default scheme of Contention Window $(\mathrm{CW})$ is not suitable in multi-hop ad-hoc networks. A dynamic window adaption scheme that is based on offer load and rate of collision in the network should be an optimal and ideal solution for the selection of $\mathrm{CW}$ at the MAC layer. The default mechanism of the contention window is not suitable for the latter two environments. So, a dynamic window adoption based on offer load/traffic and collision rate should be an ideal choice for the selection of CW.

\section{Literature Review}

In reference (Mbarushimana \& Shahrabi, 2007) author presented a review of congestion control schemes which was different from each other in the calculation of slow start threshold, manipulation of the congestion window, and bandwidth estimation in various wireless network domains. The most common and major reason for performance degradation of TCP in almost all the environments is the assumption made by TCP, that any type of packet loss is considered as congestion which is no longer valid in wireless and especially in multi-hop environments. Aside from these some other major reasons and factors that result in poor performance of TCP, Error-prone wireless channel, and handoffs in one hop wireless network. While frequent route failures, medium access contention, and breakages in multi-hop networks are the causes of performance degradation. Comparatively the performance degradation is much more severe in the case of multi-hop networks as compared to single-hop wireless environments. Finally, they critically reviewed the solution, and improvements reported by employing the proposed schemes by the community are encouraging but none of them is suitable for all the situations and meet all the difficulties and challenges mentioned. Their work concluded that some critical issues about the improvements of TCP performance and fairness are identified but still more works need to be done in the future to solve the problems and identify the hidden issues.

In reference (Jatain, 2018) investigated the effect of the varied TCP connection in ad-hoc networks using prominent ad-hoc routing protocols DSDV and AODV. The performance was evaluated based on metrics i.e. Packet Delivery Ratio (PDR), Average End to End Delay, and Average Throughput. The performance of these protocols was also weighed on based on a different number of nodes using the same evaluation parameters. From the simulation results obtained it was concluded that as the number of TCP connection was increased the throughput was also increased up to twenty connections while a decrease has been observed as the number of connections goes beyond twenty. The throughput of AODV decreased from $467 \mathrm{kbps}$ to $336 \mathrm{kbps}$ while for DSDV it was $435 \mathrm{kbps}$ to $193 \mathrm{kbps}$ as the number of connections was increased from twenty connections and nodes were varied from 20 to 200. AODV showed a decrease in packet delivery ratio as the number of TCP connections was increased while the DSDV packet delivery ratio remains constant up to 50 nodes. Delay was also increased for both protocols as the number of connections was increased but it was much higher in the instance of AODV because of the reactive nature. The average end to end delay for both the protocols increases as the number of TCP connection is increased but the AODV average end to end delay is high due to its reactive nature. The initial increase in throughput was due to the increase in the number of connections which means that the more packets will be generated but as the number of connections was raised from 20 the number of packets drop was increased, and the throughput was dropped.

In reference (Natarajan \& Mahadevan, 2017) evaluated and analyzed the performance of wellknown on-demand and table-driven protocols under TCP and UDP traffic in MANET. The protocols

Iqtidar Ali, Tariq Hussain, Kamran Khan, Arshad lqbal and Fatima Perviz

The Impact of IEEE 802.11 Contention Window on

The Performance of Transmission Control Protocol...
ADCAIJ: Advances in Distributed Computing and Artificial Intelligence Journal Regular Issue, Vol. 9 N. 3 (2020), 29-48 eISSN: 2255-2863 - https://adcaij.usal.es Ediciones Universidad de Salamanca - CC BY-NC-ND 
selected for this research study were AODV, DSR, LAR, DSDV, FSR, ZRP, and OLSR. The evaluation was based on analyzing the scalability of protocol and checking the suitability of each protocol against different network environments. A huge amount of simulation has been carried out to study different aspects of each protocol by considering a variety of simulation parameters in each scenario. The performance analysis was based on performance matrices, i.e. Routing overhead end-to-end delay, packet delivery ratio, and throughput. The simulation has been carried out for both TCP and UDP, but our focus will be on the results of TCP. All the protocols showed a delivery rate ranging from $71 \%$ to $96 \%$ for varying numbers of nodes from 100 to 200 except ZRP. In terms of throughput, AODV performs well and showed high throughput among all the other protocols in the 100 nodes scenario since AODV finds the path widely and obtain maximum connectivity in a smaller number of nodes. As the number of nodes increased from 100 to 125 and up to 200 OLSR and FSR achieved high throughput due to the fact of not using hello messages by FSR and MPR mechanism by OLSR. The overhead of LAR is higher than AODV and DSV due to concentrating the restricted flooding area to reach the destination which has the likelihood of high control overhead. In a 200 nodes scenario, the control traffic of DSR is $59.4 \%$ and $82.6 \%$ higher than DSDV and AODV respectively due to the cache route storage mechanism of DSR protocol. This was revealed from the result analysis that DSR and AODV showed optimal performance for TCP traffic but the control overhead of DSR is much higher than AODV so for TCP traffic the protocol of choice is AODV.

In reference (Sani et al., 2017) studied the factors which influence TCP performance in multi-hop wireless ad-hoc networks. They investigated the performance of TCP under three prominent routing protocols for Mobile Ad-hoc Network (MANET). Numerous factors become a factor of performance degradation for routing protocols including mobility of nodes. The routing protocols considered for evaluation were Zone Routing protocol, Dynamic source routing, and Ad-hoc on-demand Multipath Distance vector for a disaster recovery scenario. The disaster area mobility model was used in the simulation scenarios to realistically reflect the mobility of nodes in disaster recovery scenarios. The primary concern and main goal of their research study were to determine the most suitable protocol to be used in the disaster area mobility model for TCP type traffic. The simulation results revealed that TCP degrades the performance with an increase in density of the network for the selected protocols with few numbers of TCP connections. While on the contrary side the performance of TCP showed a slight increase with an increase in node density when the background connection involved is high. The overall performance of AOMDV is quite better than DSR and ZRP for TCP type traffic by keeping lower delay and maximum throughput. The performance in terms of packet delivery ratio (PDR) is best for DSR. The worst performance was showed by ZRP in all performance evaluation metrics.

In reference (Sun, 2016) tested and assessed the influence of window decrement rate on TCP in MANET. The simulation was done using the NS-2 1000*1000-meter area which lasted for 90 seconds. Packet loss, jitter, and the end-to-end delay was selected as metrics for evaluating the performance. The default decrement rate of TCP in the NS-2 simulator is 0.5 which is half of the window size, while this study varied the decrement range from 0.1 up to 0.9 . The analysis of the simulation results revealed that the alteration was negligible up-to 10 nodes but a variation in delay was detected with each decrement as the network density goes beyond 20 stations. It was noticed that the decrement rate was persuasive for dense networks. The results of delay are merely identical with jitter, which was influential for dense scenarios, with increase decrement rate the average jitter raised slightly. A reduction of $17.05 \%$ was observed in delay as the window was decremented from 0.9 to 0.1 . The pattern of jitter was also nearly the same with a smaller average decrement which was about $4.15 \%$. The packet loss

Iqtidar Ali, Tariq Hussain, Kamran Khan, Arshad lqbal and Fatima Perviz

The Impact of IEEE 802.11 Contention Window on The Performance of Transmission Control Protocol...
ADCAIJ: Advances in Distributed Computing and Artificial Intelligence Journal Regular Issue, Vol. 9 N. 3 (2020), 29-48 eISSN: 2255-2863 - https://adcaij.usal.es Ediciones Universidad de Salamanca - CC BY-NC-ND 
was unchanged with variation in window decrement because of the nature of the TCP retransmission mechanism

In reference (Assasa et al., 2018) studied the performance of dense millimeter-wave and deployed test beds containing up to eight stations. IEEE 802.11 ad millimeter-wave testbeds were deployed practically that permitted access to the parameters of lower layers of each station. The performance of the upper layer was analyzed based on the impact of lower-layer parameters. The impact of channel contention was analyzed on the buffer size of the transport layer for the first time according to their knowledge. Further, the impact of frame aggregation and the efficiency of spatial sharing were also analyzed. The protocol features of the Medium Access Control (MAC) layer and TCP in practical millimeter-wave networks were studied in this research work. A complete IEEE 802.11ad network with one AP and up to eight stations were considered on practical testbeds by using commercial off-the-shelf hardware. The inefficiencies of CSMA/CA were exacerbated by the multi-gigabit per second speed at the physical layer. Due to high error rates for large frames, frames aggregation is only favorable up to some extent. Finally, they studied delay, showed that the systematic beacon transmission time can degrade the performance by inflating the roundtrip time. It was concluded from the overall results that the characteristics of mm-wave links do neither match the behavior of wired nor traditional wireless links.

In reference (Dalal et al., 2014) presented a survey based on different types of loss mitigation techniques of the link layer in wireless ad-hoc networks. They proposed a well-established 2-state Markov model keeping different wireless errors introduced at the link layer into account. The simulations were performed by considering different settings for maximum link retransmission allowed for each frame. The simulation results indicated that performance was improved by the proposed link retransmission mechanism by limiting the losses that happened at the transmitting side of TCP. Further, they identified the adverse effect on other parameters of TCP which may cost a lot under extreme network circumstances linked with the proposed improved solution. The proposed model was evaluated by observing the effects of link retransmission schemes on multiple TCP flows contending with each other. The analysis made indicated that the TCP throughput must be maximized keeping the cost as low as possible.

In reference (Gopinath \& Nithya, 2018) worked on the MAC layer by highlighting the problem that degrades the performance of the network in multi-hop environments. They identified that the main reason for poor performance at the MAC layer is the high probability of collisions that suffers the BEB algorithm. The above problem is diminished by proposing a new efficient $\mathrm{BO}$ algorithm by using suitable integer sequences for the calculation of the new Contention window (CW) without any extra overhead and composite calculations. The collision is controlled, and the performance had been enhanced by adopting the proposed modified $\mathrm{CW}$ resetting mechanism after each successful transmission. The saturated throughput was first computed based on the analytical model developed for the proposed algorithm. Extensive simulation has been carried out using the NS-3 simulator for different packet sizes. It was concluded from the analytical and simulation analysis that the proposed backoff scheme performed optimally and enhanced the performance as opposed to the conventional BEB algorithm in terms of saturated throughput, delay packet loss ratio, and packet delivery ratio. The recommendations proposed in this research study were to consider the CW factor and other RAW parameters for developing a more effectual $\mathrm{BO}$ algorithm will be the future focus.

In reference (Jatain, 2018) evaluated numerous routing protocols techniques to investigate the behavior of these protocols regarding congestion control in MANETs. The findings have been analyzed based on performance evaluation parameters like packet delivery rate, Data error, packet drop ratio, and throughput. From the analysis of results, it was concluded that AODV achieved better results in

Iqtidar Ali, Tariq Hussain, Kamran Khan, Arshad lqbal and Fatima Perviz

The Impact of IEEE 802.11 Contention Window on

The Performance of Transmission Control Protocol...
ADCAIJ: Advances in Distributed Computing and Artificial Intelligence Journal Regular Issue, Vol. 9 N. 3 (2020), 29-48 eISSN: 2255-2863 - https://adcaij.usal.es Ediciones Universidad de Salamanca - CC BY-NC-ND 
terms of throughput, packet delivery ratio, and low delay as compared to DSR, EAODV, IRED for controlling congestion in the network. The objective of this research study was to facilitate the research community in this domain to carry out the development of enhanced new techniques.

In reference (Mahi-Rekik \& Bourenane, 2018) proposed a new scheme for the performance improvement of the IEEE 802.11 DCF mechanism. The DCF mechanism is composed of two kinds of delay i.e. Interframe sequence and bakeoff delays. In the suggested solution when a node wants to transmit data must wait for extra time which equates to at least one DIFS before beginning the process. If the medium is idle a decrement rate of one slot is applied to the bakeoff process. When the channel becomes busy the bakeoff process goes to a frozen state and was resumed after DIFS until it reached zero value. The impact of the proposed solution is studied and tested under multiple scenarios by increasing the contention level accordingly by using NS-2. The number of nodes was increased for interruption of neighboring flows. It was further revealed from the analysis of results that increase in terms of delay correspondingly to the density of the network within the flows. The major concern identified in this scheme was the ad-hoc unfairness issue in the IEEE 802.11. The gain in the delay is proportional to the number of hops in the topology since the station must wait for extra DIFS as the disruption among flows is high. Promising results were obtained for multi-hop topologies. The proposed solution works better in multi-hop scenarios by decreasing the delay by $38 \%$ while increasing the throughput by up to $81 \%$ in the specified scenarios. The two mechanisms i.e. IFS and back-off time are more likely to be present in the amended versions of IEEE 802.11 which came after 802.11. In the end, a future direction was given to simulate the different topologies through this solution by considering the other standards of 802.11 that came after $802.11 \mathrm{~b}$ standard.

In reference (Purniya \& Rai) studied TCP and some of its variants in detail with putting some light on the factors which influence TCP performance in MANETs. According to their study, the main constraints which influence and degrade the performance of TCP were high bit error rates, route failures, network partitioning, hidden and exposed node problems, the interaction between MAC and TCP, and power scarcity. Further, a simulation has been carried out to investigate the performance of TCP and its considered variants. A detailed analysis has been made based on TCP, New Reno, SACK, and Hybrid TCP variants through simulation by taking DSR as a routing protocol. The simulation has been done for the variable density of nodes and various quantity of TCP links in each scenario. NS-2 was used as a tool for simulation and the performance was assessed based on evaluation parameters, i.e. PDR, throughput, residual energy, and delay. It was observed from the simulation results that as the density of the network increased the performance of the variants was decreased. The performance of all variants was affected by the node density because of frequent path breaks increased with the low density of nodes. The overall results obtained from the simulations indicated that an increase in node density overhead and packet drop showed an increase while a decrease was observed in packet delivery ratio and throughput.

\subsection{Challenges of TCP in MANET}

A huge amount of research has been carried out to recognize the behavior of TCP and numerous solutions have been proposed for improving the performance by meeting wireless specific challenges over multi-hop wireless networks. Till now it's a hot and active research area due to the presence of wide-open problems. The primary responsibilities of TCP at the transport layer are flow control, congestion control, and error recovery, while some state-of-the-art methods include selective acknowledgments, fast retransmission, and fast recovery, etc. The main focus is on how punctually and effectively

Iqtidar Ali, Tariq Hussain, Kamran Khan, Arshad lqbal and Fatima Perviz

The Impact of IEEE 802.11 Contention Window on

The Performance of Transmission Control Protocol...
ADCAIJ: Advances in Distributed Computing and Artificial Intelligence Journal Regular Issue, Vol. 9 N. 3 (2020), 29-48 eISSN: 2255-2863 - https://adcaij.usal.es Ediciones Universidad de Salamanca - CC BY-NC-ND 
respond to congestion in the network. Some prominent features of multi-hop wireless networks which extremely decline the performance of TCP in these networks are fading and interference caused by unpredictable wireless channel, random access collision caused by susceptible shared media, frequent path breakages caused by the mobility of nodes, and the dominant hidden and exposed node problems. The challenges faced by transport layer TCP can be broadly categorized according to the network layered architecture point of view which have severe effects on its performance in the multi-hop network includes; channel errors, hidden and exposed node problems, medium contention, multipath routing, mobility, and congestion [12].

\subsubsection{Channel Errors}

The influence of channel errors on the performance of TCP in MANET is more severe because of multi-hop wireless links than that of cellular and wireless LANs where individually the last hop is wireless. In multi hops, the congestion window at the transmitter side may shrink extraordinarily due to channel errors which further results in a decreased throughput.

\subsubsection{Medium Contention, Hidden and exposed node issues}

One of the most widely used and studied Medium Access Control schemes for multi-hop ad-hoc networks is IEEE 802.11 MAC which is also been incorporated into numerous wireless testbeds and simulation packages. In this scheme, the medium is shared with neighbor nodes and every node will contend and sense the medium before transmitting. The basic and significant major issues for these networks is unfairness, exposed, and hidden station complications, and medium contention. It was also noticed by many research articles that the problem of instability and incompatibility arises due to not having proper coordination of TCP and the MAC layer further results in severe performance degradation. The two major problems which thwart one host from the other host within its transmission range are MAC layer collisions and the exposed node problems. A route failure will be triggered if a node is not able to reach the adjacent nodes several times and the source host will in turn start path discovery. Till the establishment of a route, no data will be transmitted further. For the duration of time, the TCP source has to wait, and if a time out is observed it will invoke its congestion control algorithm which results in fluctuations of TCP throughput. The situation becomes worse by the random backoff mechanism used in the MAC layer. The chance of intermediary nodes for gaining channel access is reduced due to frequent inline communication of large data packets. The nodes wait for a backoff interval and attempt over again and route failure is reported after several failed attempts (Gopinath \& Nithya, 2018).

\subsubsection{Mobility}

Mobility is another source of packet loss in multi-hop networks, due to the free movement of mobile nodes in the network topology, link breakage and route failure happen as the nodes in the network move from the transmission range of another node. As defined in the earlier section that TCP cannot differentiate the losses that occurred by route failures and congestion. TCP would react adversely and invoke the congestion algorithm to packet loss caused by route failures which will impact the performance and decrease the throughput of the network unnecessarily (Jatain, 2018; Kanellopoulos, 2019).

Iqtidar Ali, Tariq Hussain, Kamran Khan, Arshad lqbal and Fatima Perviz

The Impact of IEEE 802.11 Contention Window on The Performance of Transmission Control Protocol...
ADCAIJ: Advances in Distributed Computing and Artificial Intelligence Journal Regular Issue, Vol. 9 N. 3 (2020), 29-48 eISSN: 2255-2863 - https://adcaij.usal.es Ediciones Universidad de Salamanca - CC BY-NC-ND 


\subsubsection{Multi-path Routing}

Due to unpredictable and frequent link breakages, the lifetime of a route is very short in multihop ad-hoc networks. Routing protocols like TORA (Park \& Corson, 1997) preserve multiples routes between sending and receiving pair for reducing delay due to route reconstruction. In this type of situation, packets originated on multiple paths may not arrive in the correct order at the intended receiver. The transport layer protocol TCP is unaware of the multipath routing mechanism and will misinterpret out of order delivery of packets as a signal of congestion. The sender side will invoke the congestion control scheme like fast retransmission after receiving the duplicate ACKs generated by the receiver side which will further influence the stability of the network (Bheemalingaiah et al., 2017).

\subsubsection{Congestion}

Transmission Control Protocol is an aggressive protocol of the transport layer which tries to completely utilize the bandwidth of the network, this phenomenon leads to congestion easily due to the nature of ad-hoc networks. Moreover, variable Medium Access Control (MAC) delays and unpredictable changes in routes are some of the factors in ad-hoc networks that will affect the relationship of congestion window size and bearable data rate of the route. The computed congestion window for the old route maybe not fit for the newly discovered route and maybe too large will cause congestion because the sender will transmit according to the congestion window of the old route. Furthermore, the TCP would be affected by the buffer overflow and link contention due to an increase in network density (Ali et al., 2020; Pokhrel et al., 2017).

\section{Proposed IEEE 802.11 Contention Window System}

The first paper on this phenomenon which reported that TCP throughput is degraded due to contention window misbehavior was (Kyasanur \& Vaidya, 2003). Several misbehavior strategies were taken into account like selecting a smaller backoff range from ( 0 to $\mathrm{CW} / 4)$ having 1 fixed slot or not doubling the contention window and improved the performance up to some extent This research work simulates and evaluates TCP performance by testing the effects of CW. The performance of TCP over varying contention windows has been tested through multiple simulation scenarios. The results have been carried out two types of networks, the first one is composed of 25 nodes and the lateral one is composed of 50 nodes. Further two types of simulation scenarios are constructed for each network type. In the first phase, the maximum contention window (CWMax) varied from minor to larger window, i.e. (31 to 1023) along with the fixed minimum contention window (CWMin) i.e. CWMin=31 in this research work. In the second phase, the CWMin is varied along the fixed CWMax. The underlying channel used for accessing the medium is wireless 802.11 . RWP mobility model is used for arbitrary mobility of nodes in a specified area of $500 \mathrm{~m}$ on the $\mathrm{x}$-axis and $500 \mathrm{~m}$-axis. The routing protocol is AODV which is the most prominent in these networks according to the literature studied while the simulation has been carried out for a maximum time of 100 seconds. The simulation has run for a maximum of 50 times and average results have been taken against each simulation scenario.

\section{A. Pre-Simulation Phase}

The pre simulation phase is the preliminary stage in which all the factors are set before the actual work starts. This phase defines that how many simulation scenarios will be created and will be

Iqtidar Ali, Tariq Hussain, Kamran Khan, Arshad lqbal and Fatima Perviz

The Impact of IEEE 802.11 Contention Window on The Performance of Transmission Control Protocol...
ADCAIJ: Advances in Distributed Computing and Artificial Intelligence Journal Regular Issue, Vol. 9 N. 3 (2020), 29-48 eISSN: 2255-2863 - https://adcaij.usal.es Ediciones Universidad de Salamanca - CC BY-NC-ND 
differentiated based on simulation parameters from each other like the number of hosts in the scenario, topology of the network, mobility models, selection of protocols on different layers, simulation time, terrain size of the network, selection of performance evaluation parameters and much more beyond this. A TCL script will be written composed of the aforementioned parameters and protocols.

\section{B. Execution Phase}

The execution phase will accomplish the task of running the simulation script which will be prepared in the previous phase written in OTCL language. After executing the scripts of each simulation scenario two files will be obtained in the form of output i.e. trace and animation file. The trace (tr) file consisting of the whole events happened throughout the process of simulation for a specified amount of time such as the number of packets sent, dropped, and received etc. While the animation file contains the physical and visual layout of the network topology shown in figure 2 .

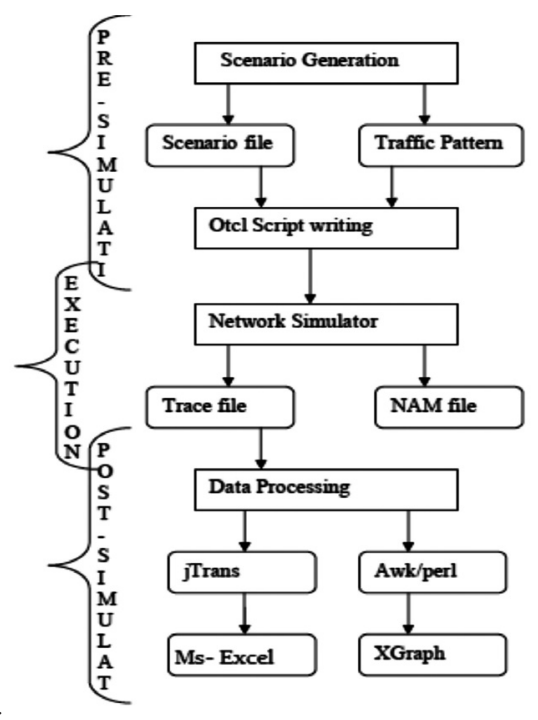

Fig 2. Phases of Simulations.

\section{Post Simulation Phase}

The last phase of the simulation process is the post-simulation phase whose purpose is to critically analyze the obtained results and get the required information from the generated files (trace files). Several techniques can be applied for getting the desired information. Perl and AWK scripts are the two common techniques used for the analysis of the results obtained from simulation while some people export the trace files to excel for analysis.

\section{Simulation Scenarios}

A huge amount of simulation will be carried out to investigate the relationship between $802.11 \mathrm{CW}$ and TCP in the MANET network. Several simulation scenarios will be tested by varying simulation parameters like the variation in the number of nodes, incorporating mobility, and variation of CW size. 
The simulation will demonstrate how TCP performance will be affected by the contention window scope in different scenarios. It is well known that the length of transmission path will increase the RTT and degrades the performance of TCP especially in terms of throughput due to higher packets drop probability and longer paths. In the first phase upper bound of the contention window (CWMax) will be increased while keeping the lower bound (CWMin) unchanged and fixed. The second phase will be consisting of changing the lower bound of the contention window (CWMin) along a fixed CWmax to observe the variation in the performance of TCP. The two phases discussed will be replicated for several scenarios by changing the density of the network in each scenario. Further, the simulation will be carried out for static and mobile scenarios. The routing protocol used will be AODV based on a literature study that is the best one for TCP traffic in mobile scenarios.

\section{E. Performance Evaluation Metrics}

The performance of protocols and algorithms after the simulation can be tested and evaluated based on some criteria i.e. evaluation metrics or parameters in the domain of networks. The performance metrics are chosen for this research study are Average throughput, Average Packet Drop, and Average End-to-End Delay.

\section{F. Average Throughput}

Throughput refers to the number of items or material passing through a system. In the jargon of networks throughput is the amount of data transferred successfully from source to destination in a network in a specified amount of time. Throughput is measured normally in bits/sec. Higher throughput denotes the effectiveness and efficiency of the network.

$$
\text { Throughput }=\sum_{\mathbf{i}=1}^{\mathbf{n}} \quad \frac{\text { Received packets i } \mathbf{x} \text { packet size }}{\text { Total simulation time }}
$$

\section{G. Average Delay}

Network Latency refers to indicate any type of delay that happens during the communication over the network. Specifically, the time elapsed by a data packet until the departure from the transmitter node in a network until the arrival at the desired destination in a network.

$$
\text { Average Delay }=\sum_{i=1}^{n} \frac{\text { (Received time i-Sent time i) }}{\text { Total data }}
$$

\section{H. Average Packet Drop}

It is the amount of the average amount of data packets that have been dropped or lost during transmission of data traveling in a network from one place to another. Drops are typically caused by transmission errors, a collision in wireless networks, and congestion in the network. The parameter is shown in table 1.

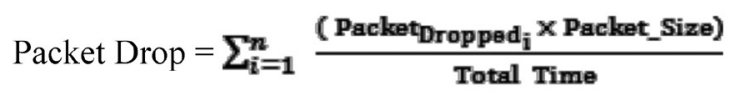

Iqtidar Ali, Tariq Hussain, Kamran Khan, Arshad lqbal and Fatima Perviz

The Impact of IEEE 802.11 Contention Window on The Performance of Transmission Control Protocol...
ADCAIJ: Advances in Distributed Computing and Artificial Intelligence Journal Regular Issue, Vol. 9 N. 3 (2020), 29-48 eISSN: 2255-2863 - https://adcaij.usal.es Ediciones Universidad de Salamanca - CC BY-NC-ND 
Table 1: Simulation Parameters.

\begin{tabular}{ll}
\hline Simulation Parameters & Values \\
\hline Simulation Tool & NS2 \\
\hline Channel & Wireless \\
\hline (MAC) Protocol & IEEE 802.11 \\
\hline Contention Window & Variable (Small, Medium, Large) \\
\hline Mobility models & Random Way Point Mobility Model \\
\hline Simulation time & 100 \\
\hline Simulation Area & 500 X 500 \\
\hline Agent & (TCP) \\
\hline Number of nodes & 25,50 \\
\hline Mobility & 0 to 10 m/s \\
\hline Traffic & File Transfer Protocol \\
\hline Routing Agent & AODV \\
\hline Packet Size & 512 Bytes \\
\hline
\end{tabular}

\section{Result and Analysis}

\subsection{Average Throughput}

Throughput refers to the number of successfully transmitted bits from the transmitter to the intended receiver in a unit time (Hussain et al., 2020; Sajjad et al.). The primary and basic characteristics of medium access control protocol of any network are to achieve higher throughput. The main causes of throughput degradation in ad-hoc networks are collisions and control overhead. As the collision occurs during the transmission the collided packets will be retransmitted to complete the communication process. The objective of the MAC protocol is to function with fewer collisions. It can be observed from figure 3, that the average throughput hardly changes for the variation in the upper bound of the contention. The throughput remains almost equal as the value of contention moved from low to high along with the fixed minimum contention window ( $\mathrm{CW}$ Min). Therefore, the range of variation in upper bound (CW Max) is not a factor of TCP degradation in terms of throughput in such networks. This illustrates that Maximum Contention Window (CW Max) have no effect or have less effect on the TCP Throughput. The upper bound of CWMax becomes too large and seems to be impossible to reach the maximum value of the upper bound the range is useless in the ad-hoc network. This is the main reason that there was no oscillation noticed for throughput by variation in the value of the upper bound of the contention window. From the observation, it was also concluded that keeping the upper bound more than 500 in a small ad-hoc scenario is useless. Comparatively, in fig 4 which is composed

lqtidar Ali, Tariq Hussain, Kamran Khan, Arshad lqbal and Fatima Perviz

The Impact of IEEE 802.11 Contention Window on The Performance of Transmission Control Protocol...
ADCAIJ: Advances in Distributed Computing and Artificial Intelligence Journal Regular Issue, Vol. 9 N. 3 (2020), 29-48 elSSN: 2255-2863 - https://adcaij.usal.es Ediciones Universidad de Salamanca - CC BY-NC-ND 
of 50 nodes, there is a variation in the results obtained for changing the value of CWMax from low to high. It was noticed that in higher traffic or heavy load scenarios more than or equal to 50 nodes the upper bound of the contention window variation has some effects. This is since the path along the topology increases as the number of nodes increased in the network. The impact is still not as it is for the minimum contention window which showed a slightly different graph in both light and heavy density scenarios. The throughput affected when the minimum contention varied from low to high the throughput changes oppositely.

\section{Nodes}

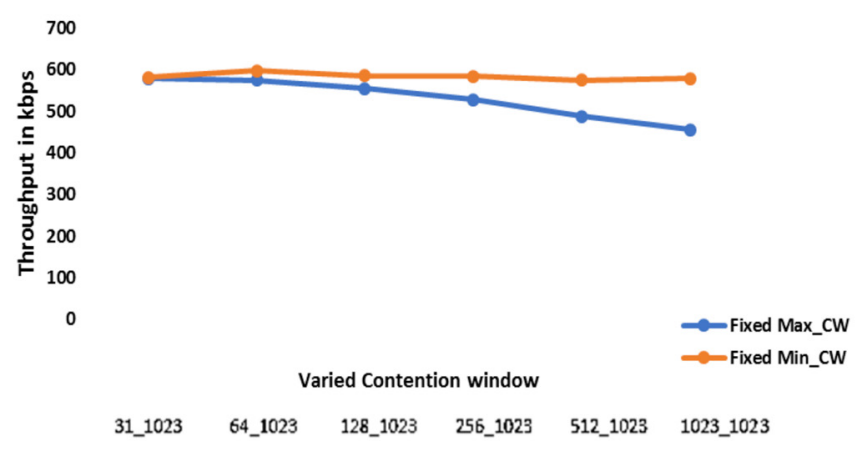

Fig 3. Average Throughput of 25 Nodes.

When the minimum contention window (CWMin) size is unreasonably large the throughput of TCP stabilizes or even showed a decrease. The reason behind this is that if the inserted delay is too large the channel will be idle too frequently to wait for a backoff or defer timer and the resources of the network are not occupied sufficiently. Therefore, it can be inferred that there is a point in the contention window size that optimizes the throughput of TCP in such networks. In figure 4 it can be observed clearly that the point of optimization is the medium contention window. Further, it can be concluded that keeping the minimal contention window low will produce high throughput especially when the density of the network is high while keeping a higher minimal contention window will show a decrease in throughput for both low and high network densities.

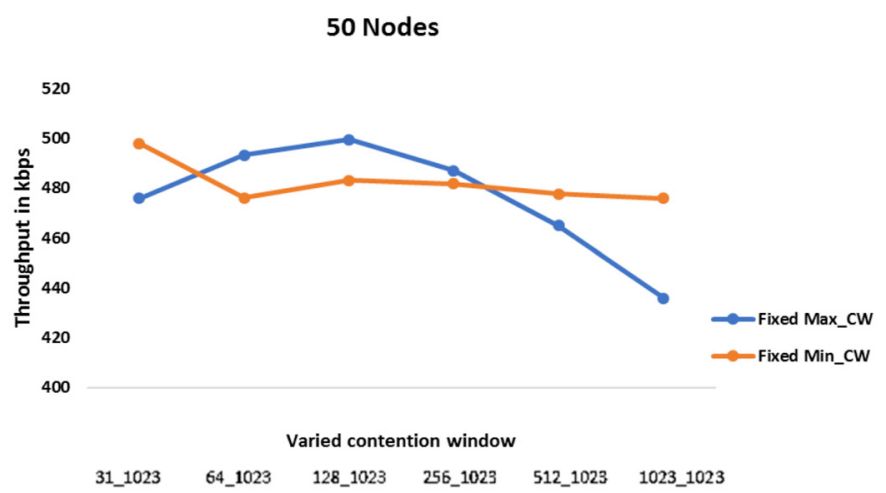

Fig 4. Average Throughput of 50 Nodes.

Iqtidar Ali, Tariq Hussain, Kamran Khan, Arshad lqbal and Fatima Perviz

The Impact of IEEE 802.11 Contention Window on The Performance of Transmission Control Protocol...
ADCAIJ: Advances in Distributed Computing and Artificial Intelligence Journal Regular Issue, Vol. 9 N. 3 (2020), 29-48 eISSN: 2255-2863 - https://adcaij.usal.es Ediciones Universidad de Salamanca - CC BY-NC-ND 


\subsection{Average Delay}

It is the amount of time elapsed for the duration of transferring data packets from the transmitter node to the intended receiver in a multi-hop ad-hoc network. The End to end delay is the combination of three types of delays i.e. propagation delay, transmission delay, and processing delay of packets. The contention window is the total 'quantity of time distributed into slots. It is doubled up with every collision that happened exponentially. In this work values of the contention, a window is assigned statically ranging from low to high, identify its impact of TCP performance, and choose the suitable window where the performance is optimal.

\section{Nodes}

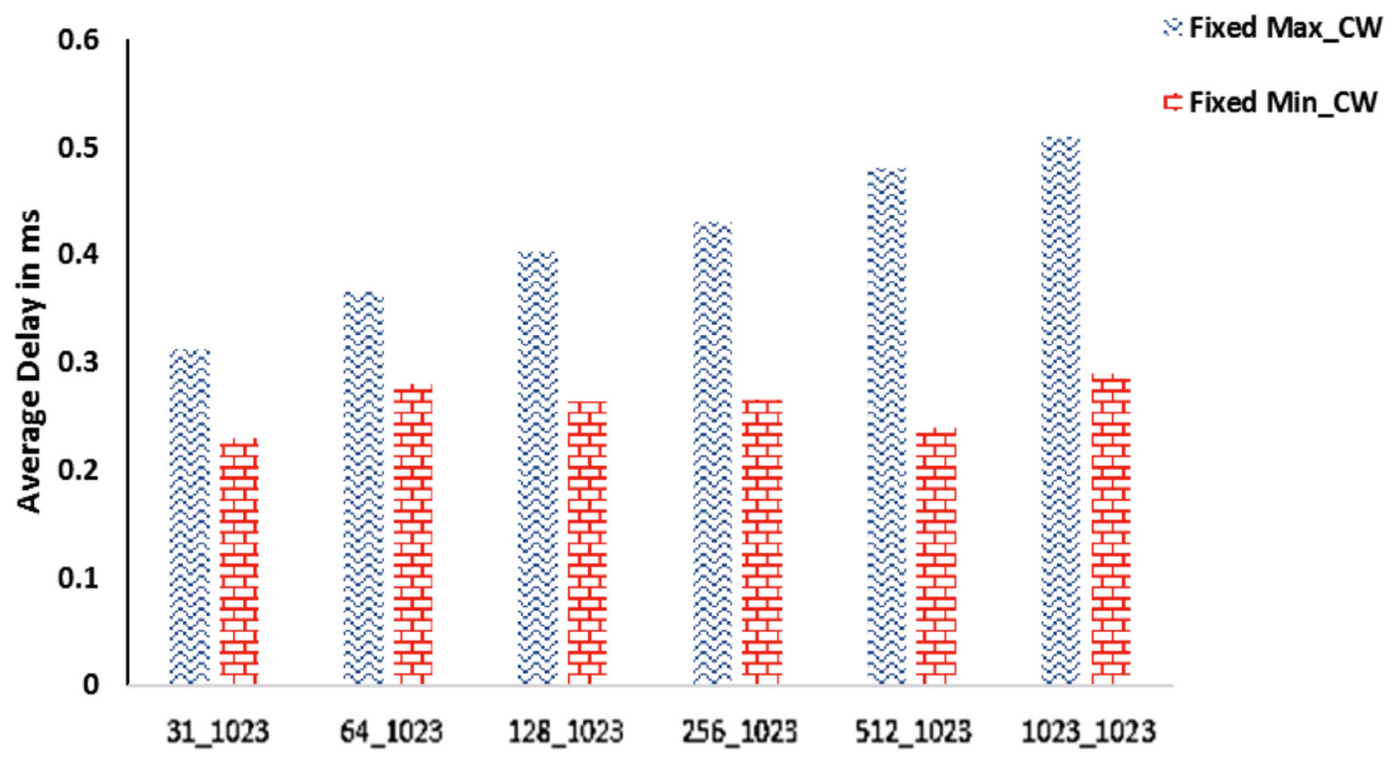

Fig 5. Average Delay of 25 Nodes.

Figure 5 and figure 6 reveal the average delay of varied minimal and maximal contention windows for 25 and 50 nodes respectively. It was observed from both the figures as we discussed earlier in the discussion of throughput in detail that the upper bound of the contention has no effect on TCP performance in a small network and a negligible impact in high nodes density. The same is the case here in terms of average delay. While the results of CWMin go parallel with the increase in minimum contention window as the value of CWMin increases so as the increase was noticed in the average delay. This is since enlarging the contention window size eventually increases the backoff time and the differing time which inserts more delay among the outgoing data packets. Further justification is that if the inserted delay becomes too large then the channel will be idle more frequently to wait for a defer or back off timer, so the network resources are not sufficiently occupied. With the increase in network density in the second simulation scenario, the average delay of varying contention window increases

Iqtidar Ali, Tariq Hussain, Kamran Khan, Arshad lqbal and Fatima Perviz

The Impact of IEEE 802.11 Contention Window on The Performance of Transmission Control Protocol...
ADCAIJ: Advances in Distributed Computing and Artificial Intelligence Journal Regular Issue, Vol. 9 N. 3 (2020), 29-48 eISSN: 2255-2863 - https://adcaij.usal.es Ediciones Universidad de Salamanca - CC BY-NC-ND 
more due to the reason that adding more nodes to the scenario can result in long paths which will take more time which is directly effectual on the average delay of the network.
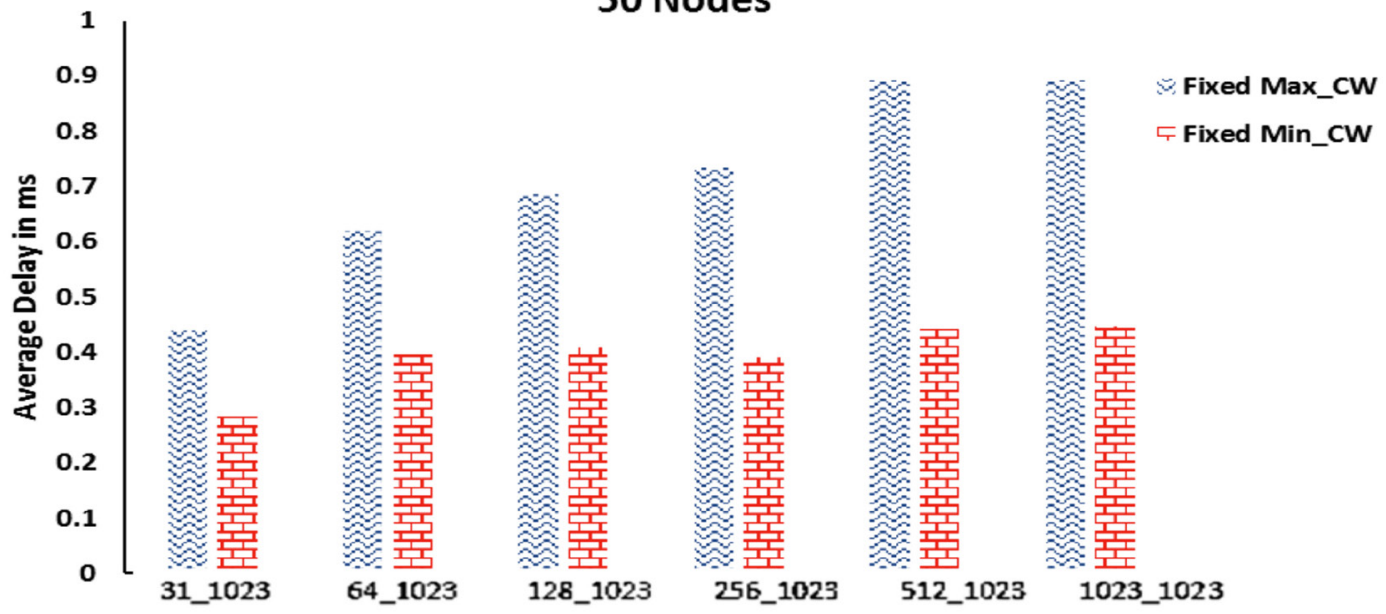

Fig 6. Average Delay of 50 Nodes.

\subsection{Average Packet Drop}

It is the amount of data packets that are initiated accurately from the transmitter side and are not delivered to the desired receiver node. The unsuccessful reception of data at the destination may come from several reasons like link failure, congestion, and contention, interference and collisions, etc. It can be observed from figure 7 and figure 8 that finally a slight difference can be seen for the upper bound of the contention window. Keeping the upper bound too small i.e. CWMin=CWMax can drop more data. We can see that 1070 has been dropped which is comparatively high which is for when the size of CWMin=CWMax. Keeping the upper bound to small is not a good choice in such networks. The drop rate becomes much in larger network scenarios for keeping a very small value for CWMax the number of packets drops range above 1300 packets.

The reason behind the high packet drop in high network density scenario is that the number of hops will be high, and the low value doesn't fit to deliver data accurately and timely due to the interference and collision between data flows and acknowledgment flows. A larger contention will be needed to absorb such network degradation.

Iqtidar Ali, Tariq Hussain, Kamran Khan, Arshad lqbal and Fatima Perviz

The Impact of IEEE 802.11 Contention Window on The Performance of Transmission Control Protocol...
ADCAIJ: Advances in Distributed Computing and Artificial Intelligence Journal Regular Issue, Vol. 9 N. 3 (2020), 29-48 eISSN: 2255-2863 - https://adcaij.usal.es Ediciones Universidad de Salamanca - CC BY-NC-ND 


\section{Nodes}

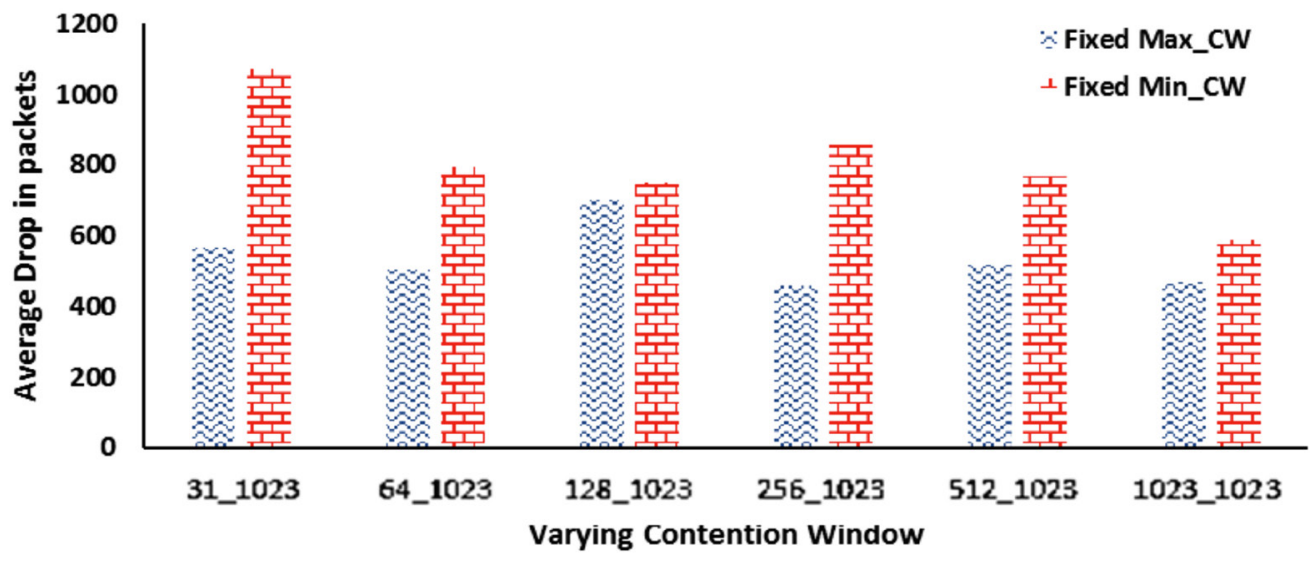

Fig 7. Average Packet drop of 25 Nodes.

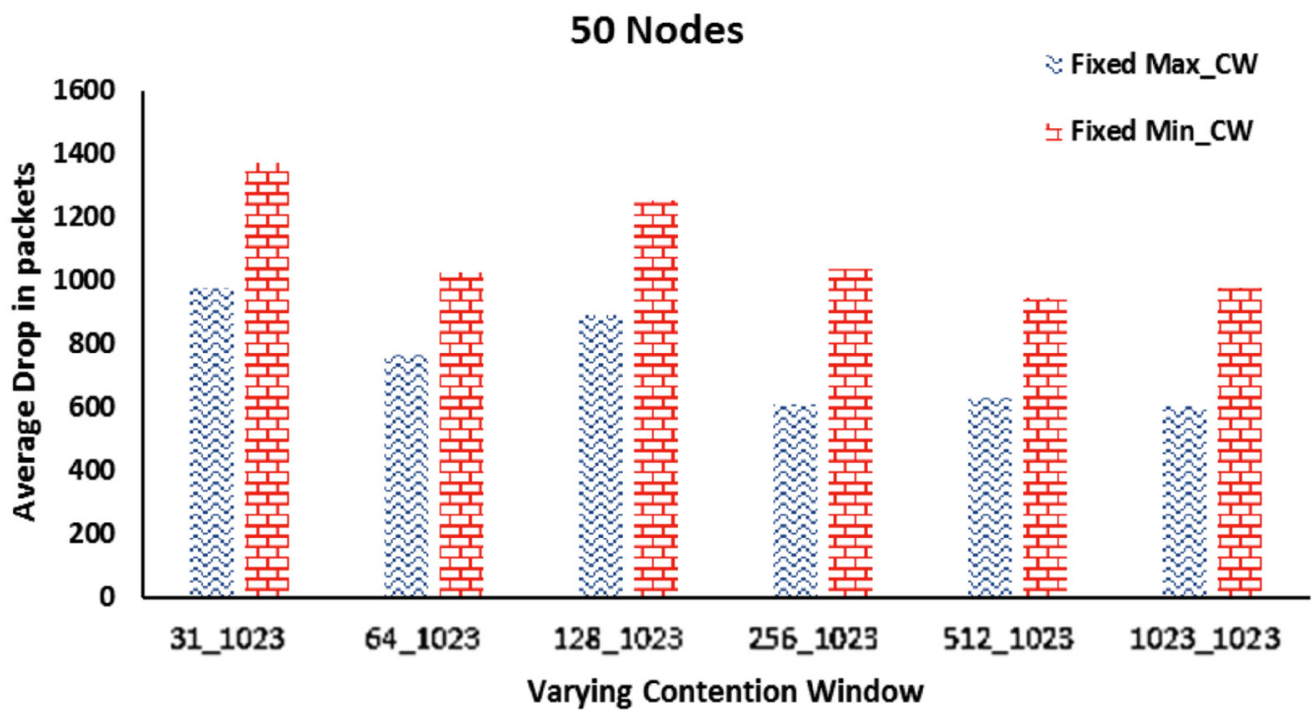

Fig 8. Average Packet drop of 50 Nodes.

ADCAIJ: Advances in Distributed Computing and Artificial Intelligence Journal Regular Issue, Vol. 9 N. 3 (2020), 29-48 eISSN: 2255-2863 - https://adcaij.usal.es Ediciones Universidad de Salamanca - CC BY-NC-ND 


\section{Conclusion}

Back in the 1990s, the first routing protocol designed for mobile ad-hoc networks was based on the number of hops as a metric for the selection of the route. The subsequent proposal was attempted by changing the routing metric and considered the alternative methods by opting for the metrics such as link reliability, available bandwidth, and mobility of nodes as the parameters for the selection of the optimal route. Later followed by considering route lifetime regarding mobility and duration of battery life as the metrics for selection of routes in ad-hoc networks. Recent works in the ad-hoc domain and more specifically for the improvement of TCP protocols research community have focused their efforts on providing a scheme for the improvements of MAC layer parameters. One major and mutual feature of performance degradation of TCP in such networks is the unstable condition and lower layer parameters which are completely unknown from the upper layers. These lower layer parameters affect the performance of TCP up to some extent and can also degrade severally in some cases. The focus of this research study is also parameters of the MAC layer i.e. Checking the impact of upper and lower bound of the contention on TCP performance in such networks. After carrying huge simulation and varying some parameters it was concluded that to enhance the performance and to find the optimal point of performance the initial minimum contention window CW Min is the key. If the initial CW Min is set to the optimization point like the optimization point discussed in the result discussion section of average throughput the system can deliver high performance and good results. For this purpose, a new way should be adapted to introduce the dynamic computation of the CW Min to an optimal value. From the analysis of the overall results of this research study, it can be concluded that in future performance enhancement of TCP solutions the dynamic computation of optimal value for the lower bound of contention window should be considered. The minimal contention window (CW Min) is of great importance to improve the performance of upper-layer protocols in Mobile Ad-hoc Networks. The size of the contention window size should be increased or decreased according to the traffic load and rate of collisions in the network. This will minimize the internal and external collisions among different flows up to some extent.

The recommendation or future proposal should be that the present work should be carried out in other domains like Wireless Sensor Networks, Vehicular Ad-hoc Networks to analyze the potential benefits provided by this work. Further, it would be more interesting and of a great extent if the results would be showed for real implementations using a testbed environment.

Authors' contributions: All the authors contributed to this research. The order of authors in this manuscript is maintained depending on the level of contributions they made in this research.

Conflicts of Interest: The authors declared that they have no conflicts of interest.

\section{References}

Ali, I., Hussain, T., Perviz, F., \& Hussain, A. (2020). Analysis of TCP Congestion Control Queuing Mechanism and Investigation for High Throughput and Low Queuing Delay (2516-2314).

Assasa, H., Saha, S. K., Loch, A., Koutsonikolas, D., \& Widmer, J. (2018). Medium access and transport protocol aspects in practical 802.11 ad networks. 2018 IEEE 19th International Symposium on" A World of Wireless, Mobile and Multimedia Networks"(WoWMoM),

Iqtidar Ali, Tariq Hussain, Kamran Khan,

Arshad lqbal and Fatima Perviz

The Impact of IEEE 802.11 Contention Window on

The Performance of Transmission Control Protocol..
ADCAIJ: Advances in Distributed Computing and Artificial Intelligence Journal Regular Issue, Vol. 9 N. 3 (2020), 29-48 eISSN: 2255-2863 - https://adcaij.usal.es Ediciones Universidad de Salamanca - CC BY-NC-ND 
Babu, N. R., \& Geethanjali, N. A ROBUST RETRANSMISSION SCHEME FOR RESOLVING HIDDEN NODE PROBLEM IN IEEE 802.11 WLANS.

Banerji, S., \& Chowdhury, R. S. (2013). On IEEE 802.11: wireless LAN technology. arXiv preprint arXiv:1307.2661.

Bheemalingaiah, M., Naidu, M., Rao, D. S., \& Moorthy, P. S. (2017). Survey of Routing Protocols, Simulation Tools and Mobility Models in Mobile Adhoc Networks. International Journal of Innovations \& Advancement in Computer Science (IJIACS) ISSN (2017), 2347-8616.

Bhople, M. A. D., \& Tijare, P. (2011). An analysis of ADTCP, I-ADTCP and Cross-Layer Based Protocol for Improving Performance of TCP in Mobile Adhoc Network. International Journal Of Computer Science And Applications, 4(2).

Chu, B. (2005). Improving IEEE 802.11 performance with power control and distance-based Contention window Selection Citeseer].

Dalal, P., Sarkar, M., Dasgupta, K., \& Kothari, N. (2014). Link layer correction techniques and impact on TCP's performance in IEEE 802.11 wireless networks. Communications and Network, 2014.

Gopinath, A. J., \& Nithya, B. (2018). Mathematical and simulation analysis of contention resolution mechanism for IEEE 802.11 ah networks. Computer Communications, 124, 87-100.

Hussain, T., Rehman, Z. U., Iqbal, A., Saeed, K., \& Ali, I. (2020). Two hop verification for avoiding void hole in underwater wireless sensor network using SM-AHH-VBF and AVH-AHH-VBF routing protocols. Transactions on Emerging Telecommunications Technologies, 31(8), e3992.

Jatain, R. (2018). Review on Congestion Control in MANET. International Journal of Scientific Research in Computer Science, Engineering and Information Technology, 3(3).

Kanellopoulos, D. (2019). Congestion control for MANETs: An overview. ICT Express, 5(2), 77-83.

Khawas, U., \& Gautam, K. (2017). Impact of Multiple TCP Connection and Increment of Number of Nodes in Mobile Ad-Hoc Wireless Network. International Journal of Computer Applications, 168(13).

Kyasanur, P., \& Vaidya, N. H. (2003). Detection and Handling of MAC Layer Misbehavior in Wireless Networks. DSN,

Mahi-Rekik, L. S., \& Bourenane, M. (2018). IEEE 802.11 DCF Improvement: Waiting DIFS while Waiting Back-off. International Journal of Computing and Digital Systems, 7(04), 215-223.

Mbarushimana, C., \& Shahrabi, A. (2007). Comparative study of reactive and proactive routing protocols performance in mobile ad hoc networks. 21st International Conference on Advanced Information Networking and Applications Workshops (AINAW'07),

Natarajan, K., \& Mahadevan, G. (2017). Evaluation of seven MANET Routing Protocols using Scalability Scenario. Int J Comp Sci, 6(2), 131-141.

Nithya, B., Mala, C., \& Sivasankar, E. (2014). A novel cross layer approach to enhance QoS performance in multihop adhoc networks. 2014 17th International Conference on Network-Based Information Systems,

Park, V. D., \& Corson, M. S. (1997). A highly adaptive distributed routing algorithm for mobile wireless networks. Proceedings of INFOCOM'97.

Pokhrel, S. R., Panda, M., \& Vu, H. L. (2017). Analytical modeling of multipath TCP over last-mile wireless. IEEE/ACM Transactions on networking, 25(3), 1876-1891.

Iqtidar Ali, Tariq Hussain, Kamran Khan,

Arshad lqbal and Fatima Perviz

The Impact of IEEE 802.11 Contention Window on

The Performance of Transmission Control Protocol..
ADCAIJ: Advances in Distributed Computing and Artificial Intelligence Journal Regular Issue, Vol. 9 N. 3 (2020), 29-48 eISSN: 2255-2863 - https://adcaij.usal.es Ediciones Universidad de Salamanca - CC BY-NC-ND 
Purniya, R., \& Rai, D. INTERNATIONAL JOURNAL OF ENGINEERING SCIENCES \& RESEARCH TECHNOLOGY A COMPARATIVELY ANALYSIS OF VARIOUS MANET BASED THROUGHPUT ENHACEMENT TECHNIQUES.

Sajjad, M., Saeed, K., Hussain, T., Abbas, A., Khalil, I., Ali, I., \& Gul, N. Impact of Jelly Fish Attackonthe Performance of DSR Routing Protocol in MANETs.

Sani, A. M. I. P. H., Newaz, S. S., Wani, S. M. A., Wan, A. T., \& Jahan, S. (2017). Tcp performance evaluation under manet routing protocols in disaster recovery scenario. 2017 4th International Conference on Advances in Electrical Engineering (ICAEE),

Sun, W. (2016). On Medium Access Control for Vehicular Communication Over Device-to-device Links: Radio Resource Management and Network Synchronization. Communication Systems Group, Department of Signals and Systems, Chalmers.

Zhai, H., Wang, J., Chen, X., \& Fang, Y. (2006). Medium access control in mobile ad hoc networks: challenges and solutions. Wireless Communications and Mobile Computing, 6(2), 151-170. 